Hybrid circuits for the CMS tracker upgrade frontend electronics

This content has been downloaded from IOPscience. Please scroll down to see the full text. 2013 JINST 8 C01041

(http://iopscience.iop.org/1748-0221/8/01/C01041)

View the table of contents for this issue, or go to the journal homepage for more

Download details:

IP Address: 137.138.125.163

This content was downloaded on 08/07/2014 at 09:07

Please note that terms and conditions apply. 
TOPICAL WORKSHOP ON EleCtRonics fOR PARTICLE Physics 2012, 17-21 SEPTEMBER 2012, OXFORD, U.K.

\section{Hybrid circuits for the CMS tracker upgrade frontend electronics}

\section{G. Blanchot}

CERN,

Route de Meyrin, CH-1211 Genève 23,Switzerland

E-mail: georges.blanchot@cern.ch

ABSTRACT: The upgrade of the CMS tracker at the HL-LHC requires the design of new front-end modules to cope with the increased luminosity and to implement L1 trigger functionality. The new modules under development are based on high density hybrid circuits with new flip-chip frontend ASIC, and are wire bonded to strip sensors and connected to a service board for the data transmission. The suitability of different substrate technologies considered for the design of the hybrids is discussed, aiming for a cost effective and reliable manufacturability of the CMS tracker modules.

KEYWORDS: Detector design and construction technologies and materials; Front-end electronics for detector readout 


\section{Contents}

1 Introduction to the CMS tracker upgrade 1

2 Double sided strip module 2

3 Strip-Pixelated Strip module $\quad 4$

4 Build-up substrates $\quad 5$

5 Flexible substrates $\quad 6$

$\begin{array}{lll}6 & \text { Conclusions } & 7\end{array}$

\section{Introduction to the CMS tracker upgrade}

In view of the High Luminosity LHC, the CMS tracker needs to be upgraded to match the new physics requirements. This yields to new requirements for the detector modules of the new tracker that will feature higher granularity, lower mass, as well as the ability to correlate locally the signals from two silicon sensors:

- The higher rate of events drives the need for an increased granularity of the tracking detector.

- The increased luminosity, up to $3000 \mathrm{fb}^{-1}$, pushes the radiation hardness requirements for the Silicon sensors, for the front-end ASICs, but also for the mechanical components and the electronic substrate materials.

- The material budget in the CMS tracker at the LHC is mainly contributed by services in the detector volume such as power cables and cooling. The increased granularity at the HLLHC must be linked to a reduction of the material budget contributed by the tracker modules and its services. This is obtained using new CMOS technologies for the front-end ASICs, a low power Giga Bit Transmitter (LP-GBT), all combined with the use of DC-DC converters that result in a more efficient power delivery, hence less cables, less heat, less cooling and therefore less mass.

- The rejection of low momentum tracks, and the track correlation between closely spaced sensors enable the Level 1 trigger information at the tracker front-end for a more efficient use of the data required bandwidth.

The new tracker is based on different types of double sided modules: a double sided strip sensor module for the outer tracker regions and the endcaps, a strip and pixelated strip sensor module for the inner barrel regions, and an alternative 3D pixelated strip modules for the inner and outer tracker regions [1]. A module typically consists of silicon sensors (segmented in pixels or 

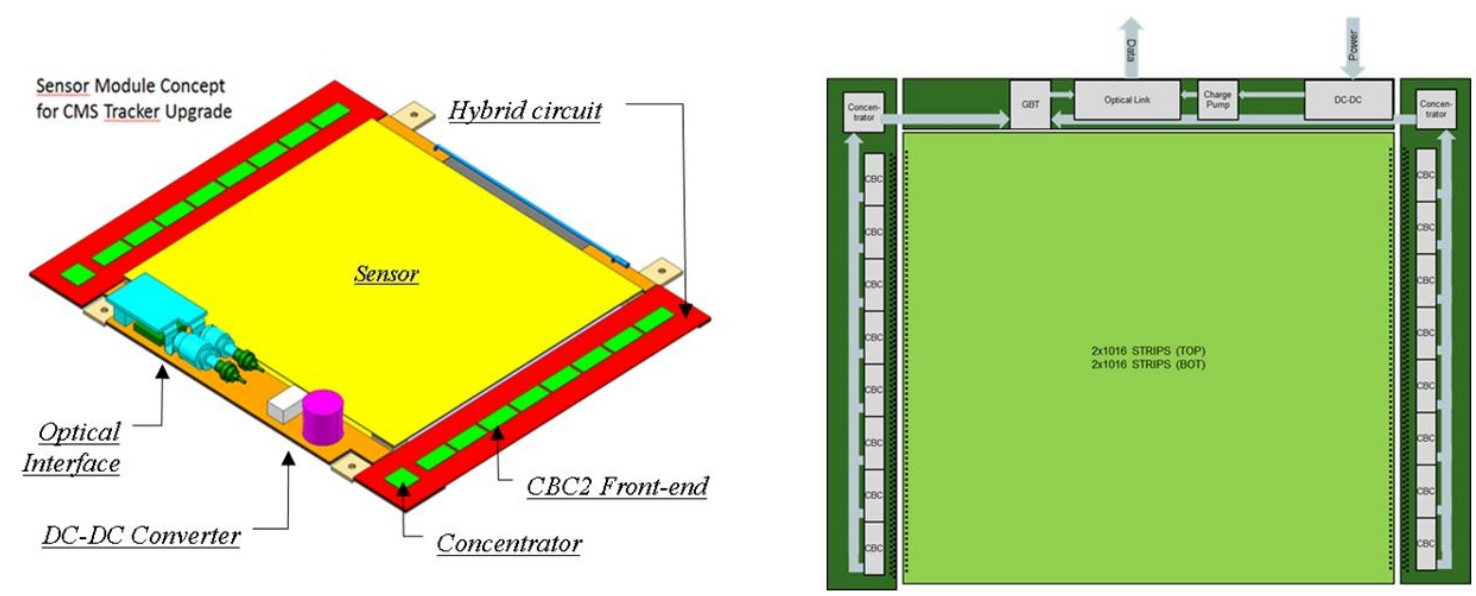

Figure 1. Double sided strip module with two readout hybrids and one service hybrid (left) and its corresponding block diagram (right).

strips), front-end hybrid circuits carrying the readout ASICs, service hybrids carrying the auxiliary electronics, and a lightweight mechanical assembly providing structural support and efficient thermal contacts to remove the heat produced by the electronics and the silicon sensors. The assembled modules must work reliably in a harsh environment featuring high radiation levels (up to $2 \times 10^{15} n_{\mathrm{eq}} / \mathrm{cm}^{2}$ integrated particle fluence), strong magnetic field (4T) and large temperature variations (from $25^{\circ} \mathrm{C}$ to $-35^{\circ} \mathrm{C}$ ), with no possibility of maintenance and repairs over an expected time frame of more than a decade.

This paper refers to the double sided strip and to the strip and pixelated strip hybrid circuits developments.

\section{Double sided strip module}

The double sided strip modules focus on the tracking at the outer barrel and the endcap regions of the tracker. Their sensing area is built on two strip sensors that are combined on the top and bottom layers of a module, enabling the low momentum tracks discrimination. Each silicon sensor is made of 1016 left side strips and right side strips that are $50 \mathrm{~mm}$ long over a pitch of 90 microns. The two overlapped sensors cover therefore an area of $92 \mathrm{~mm}$ times $100 \mathrm{~mm}$ that are read out by two hybrid circuits on the left and right hand sides of the module (figure 1).

The rejection of low momentum tracks within a module is based on the correlation between top and bottom sensor hits that create the formation of stubs (figure 2). The identification of stubs requires that the top and bottom strips get connected to common front-end ASICs that will perform this identification, but it requires also the interconnection of input strips on neighboring front-end ASICs to tag the stubs across them. The low momentum stubs are immediately rejected by the front-end ASICs while the high momentum stubs will contribute to the trigger formation, hence resulting in the event acquisition through a pipeline within the ASICs. The tracking processing is performed by the CMS Binary Chip 2 (CBC2) front-end ASICs [2].

Two hybrid circuits are required to interface the sensor strips with their corresponding CBC2 readout ASICs. These hybrid circuits collect the strip signals from the top and bottom sensors 


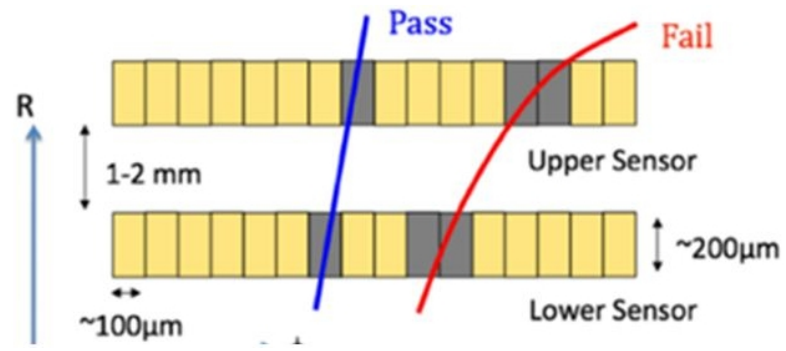

Figure 2. Low momentum rejection within a double sided strip module.

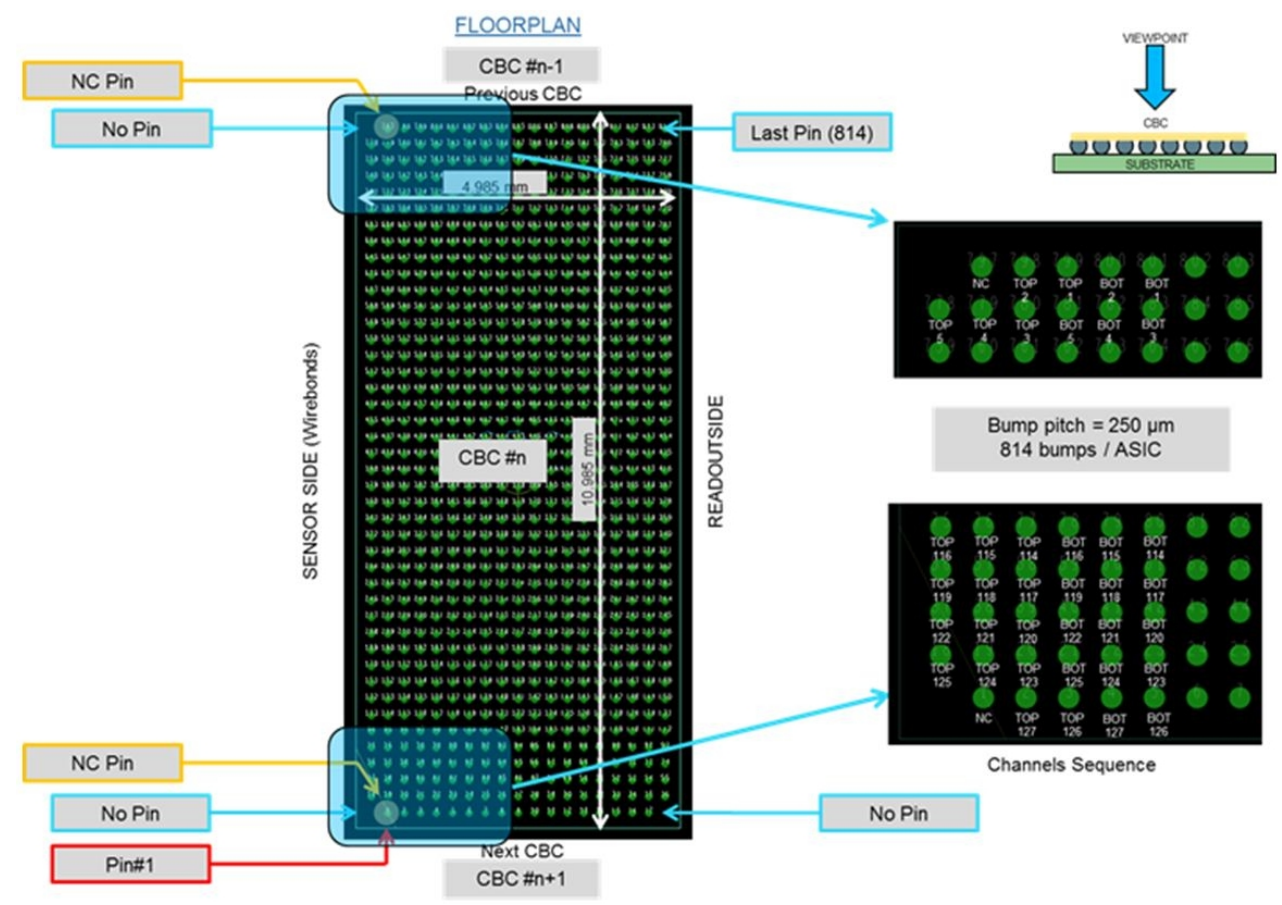

Figure 3. CBC2 flip chip footprint pattern.

on their respective sides. The $\mathrm{CBC} 2$ front-end ASICs provide 254 readout channels, split in two blocks of 127 inputs for the top and the bottom sensors, enabling in this way the identification of stubs. Eight ASICs are hence required to fully read out the sensors on each hybrid circuit. They are located in line along the hybrid circuit, almost closely abutted. The channels located in the vicinity of two dice are propagated between them along the abutted edges to perform the stub correlation within two front-end dice.

The front-end CBC2 dice are implemented on a C4 flip chip technology with a $250 \mu \mathrm{m}$ pitch (figure 3). This technology reduces the wire bonding dead space to connect the die on the hybrid. It allows also abutting the dice along the strip axis, resulting in this way in a less sensitive noise pick up path. Less inductive parasitic connections are also provided, being this of particular interest for the power distribution into the ASIC. The digital path of the assembled hybrids can also be fully tested with any wire bonding process on the dice, by means of dedicated test pads conveniently located on the hybrid itself. 


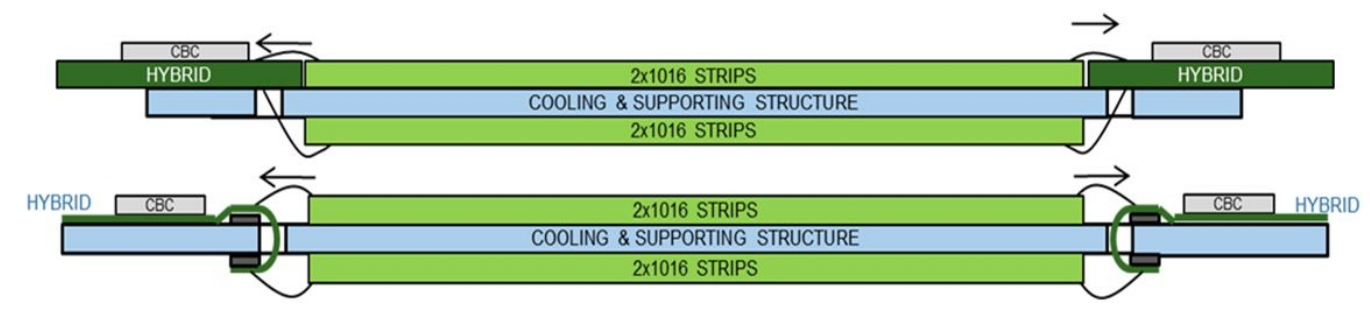

Figure 4. Module cross sections using a rigid build-up substrate (top) or a flexible foil substrate (bottom).

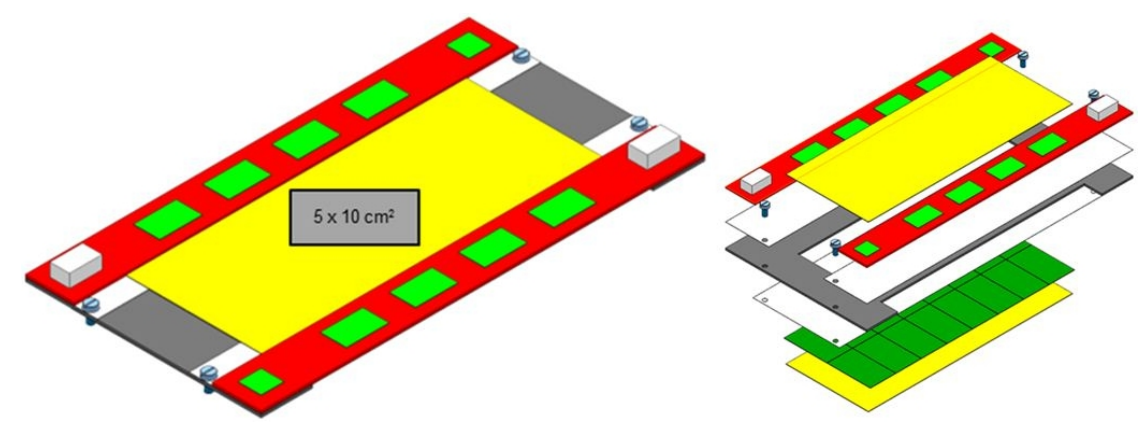

Figure 5. Strip-Pixelated strip module.

The CBC2 strip inputs are located on the six first rows of the die. This enables a straight connection from the die to the sensor wire bond pads at a minimal distance typically using two routing layers only.

High density routing is required to connect the bump array of the dice with the sensor wire bonding array. Typical tracks widths of $25 \mu \mathrm{m}$, spacing rules of similar values, and microvias drilled at $50 \mu \mathrm{m}$ are required.

Rigid build up substrates typically used for chip packaging [3] meet the above requirements (figure 4). The bottom side wirebonds reach the hybrid bottom side through a window opening in the support structure.

Flexible polyimide foils typically used in medical applications and in recent physics detector front-end systems like the Origami module at the Belle II SVT detector [4] meet also the above requirements (figure 4). In this case, the foil needs to be wrapped around the support window and glued on it. The bonding areas need a rigid reinforcement to maintain the bonding pads flatness.

\section{Strip-Pixelated Strip module}

The strip and pixelated strip modules focus on the tracking at the inner barrel regions of the tracker. The availability of pixelated sensing areas in this region brings valuable information on tracks along the $\mathrm{Z}$ axis that is of primary interest to contribute in the trigger formation. A strip sensor made of two times $25 \mathrm{~mm}$ long strips on the top layer is correlated with a $1.5 \mathrm{~mm}$ long pixelated strip sensor on the bottom layer at the same pitch of typically 100 microns (figure 5).

The pixelated strip sensor on the bottom of the module is bump bonded on a matrix of pixel front-end ASICs that cover the pixel array. On the other hand the hybrid circuits still contain strip front-end ASICs that first collect the analogue data from the top side strip sensor, then sends its 


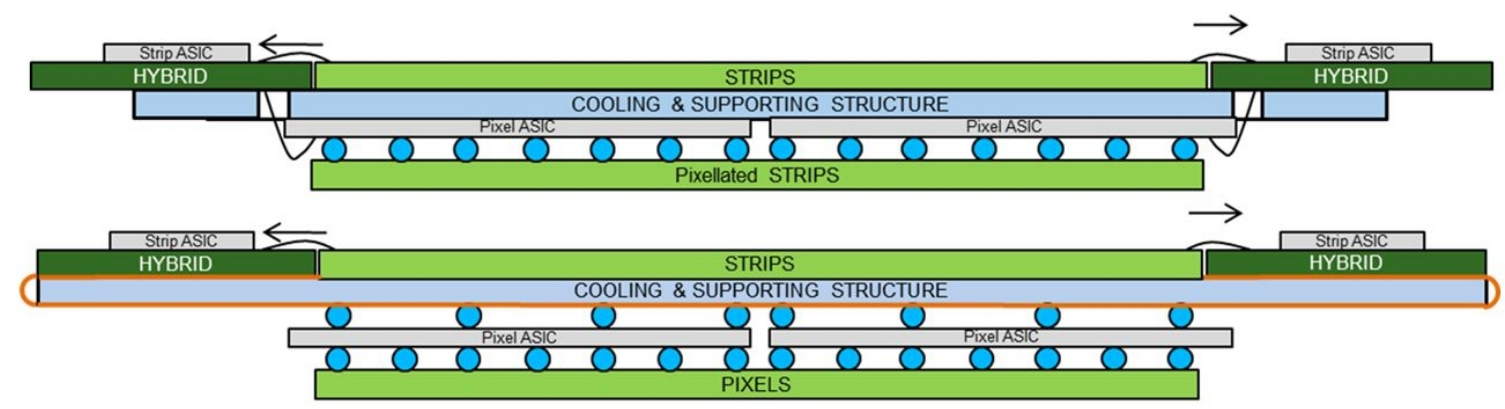

Figure 6. Strip-Pixellated strip module cross section using a rigid substrate (top) or a flexible foil (bottom).
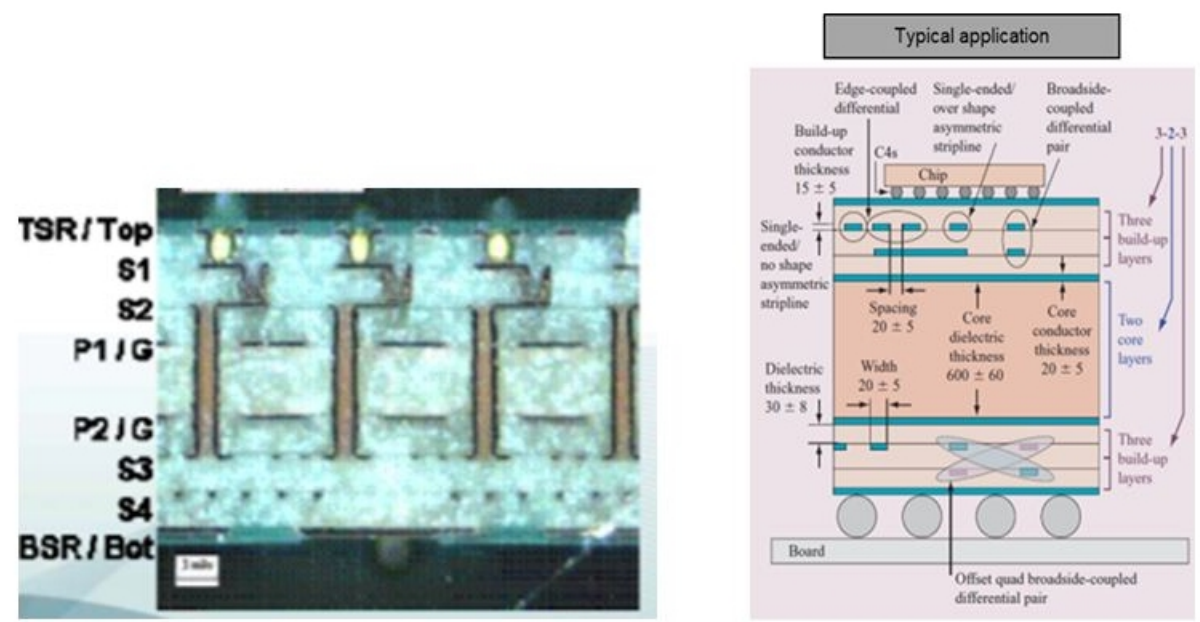

Figure 7. Typical built-up substrate with one core layer and six additional build-up layers (left) and a typical application for BGA packaging (right).

digitized information towards the pixel ASIC that will finally correlate it with the pixelated strip information. The correlated information is returned to the hybrid circuits and is propagated towards the optical interface.

Rigid build-up substrates or flexible polyimide foils are both applicable to this module as well (figure 6). Rigid circuits are compatible with a pixel ASIC bump bonded on the pixel sensor and glued straight on the support structure; they are wire bonded directly to the bottom side of the hybrid circuit. A flexible circuit can be used instead, removing the window in the supporting structure. The pixel ASIC can eventually be bump bonded on its back side using through silicon vias: in this case a better power delivery can be assured to the ASICs, and the electrical interconnection on both sides becomes possible. The development of through silicon vias on ASICs are currently under study at CERN by means of the $3 \mathrm{~T}$ project.

\section{Build-up substrates}

The need for packaging high pin count array components in the industry pushed the development of new substrate and printed circuit board technologies (figure 7) [3]. The increasing ball grid array or even flip chip dice bump densities have set the need for much thinner track width or spacing 
distances and a much better flatness and surface finish at the substrate level. New materials were developed, that are combined with direct image printing or laser drilled blind or buried microvias.

Multilayer circuits can be manufactured as a build-up substrate that consists of a core multilayer stack used for power and ground planes and for mid density routing, with additional layers that are laminated on top of it, on both sides, for high density routing. The flip chip dice are mounted on it, and microvias and fine width tracks allow the connection of the dice bumps to the wire bond pads.

Build up substrates are known for the BGA packaging of flip chip dice and for system in packages. Several suppliers have been identified for this type of product, meeting the required track width, spacing, microvia size and number of layers required for the CMS tracker hybrids. For this application and to cope with the required mass reduction, only organic or plastic based materials are considered, aiming for the thinnest stack geometry. Thin multilayer organic substrates, with typical stack thicknesses of less than $500 \mu \mathrm{m}$ in a six layer topology, using track widths and spacing constraints of less than $30 \mu \mathrm{m}$ and laser vias of less than $50 \mu \mathrm{m}$ drills over pads of $110 \mu \mathrm{m}$ diameter. This enables the connectivity of flip chip dice with a bump pitch of $250 \mu \mathrm{m}$. The radiation hardness of organic materials has also been successfully evaluated at CERN up to the doses required for the trackers.

Organic build-up substrates have a coefficient of thermal expansion (CTE) between $16 \mathrm{ppm} /{ }^{\circ} \mathrm{C}$ and $19 \mathrm{ppm} /{ }^{\circ} \mathrm{C}$ that do not match the one of silicon, that is typically of $3 \mathrm{ppm} /{ }^{\circ} \mathrm{C}$. Standard applications related to single chip packaging overcome this issue underfilling the dice to bring a better mechanical stability of the assembly. However the CMS tracker hybrid circuits will contain eight dice and more than one thousand bond pads along a distance of $92 \mathrm{~mm}$. This requirement, when combined with the thermal cycling range imposed by the low temperature operation of the hybrids $\left(+20^{\circ} \mathrm{C}\right.$ to $\left.-30^{\circ} \mathrm{C}\right)$ will result in a non-negligible thermal expansion/contraction of up to 100 microns, that is equivalent to the bond pads pitch and half the bump pad pitch on the ASICs. This effect is even more critical during the soldering process of the dice on the substrate, where larger temperatures are required. All the dice will be underfilled to reinforce the mechanical stability and extensive mechanical tests will be performed on sample designs for the development of the CMS tracker hybrids.

\section{Flexible substrates}

Medical applications have also an increasing demand for the dense integration of electronic systems. Flexible polyimide circuits provide very low mass, very dense circuits typically used in this type of products that can be suitable for the CMS tracker hybrids too (figure 8). Several suppliers have been also identified in this area, meeting the CMS tracker hybrids requirements.

The production of this specific dense circuitry is based on liquid polyimide that is spinned on a carrier substrate; the circuit is applied by means of direct image laser printing that allows reaching very small tracks and spacing constraints, the routing density being very high. The flexible polyimide foils thickness can be as low as ten microns with a copper thickness of less than seven microns only: multilayer flexible structures can quickly lose their flatness as the number of layers increases, this being a limiting factor for the total number of layers. The maximum number of layers currently cannot exceed four layers. It must be noted also that the bottom layer is used as 

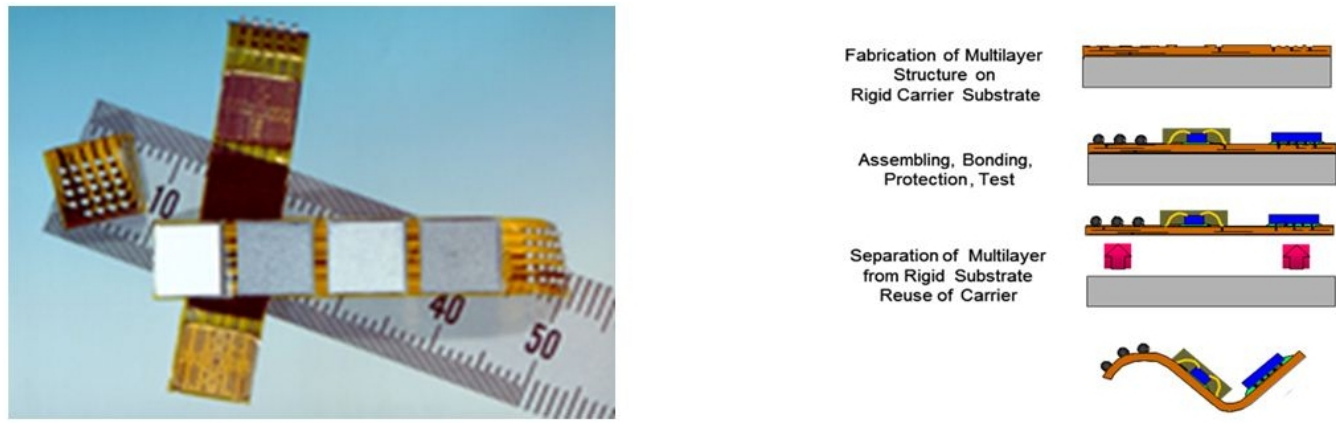

Figure 8. Medical flexible polyimide circuit (left) and fabrication method (right).

interface with the release coating, hence it can't be metalized. The CMS tracker hybrid design must take this issue in consideration.

The very thin polyimide and copper coating layers are of particular interest to cope with the front-end electronics mass reduction. It is also noticeable that the specific polyimide material used for this type of product presents a coefficient of thermal expansion close to $3 \mathrm{ppm} /{ }^{\circ} \mathrm{C}$ in the $\mathrm{XY}$ plane that matches very well the one of Silicon. The thermal cycling ranges would not cause any mechanical stress on the bumped dice, and the wire bonding pad expansion would become negligible even on the scale of the CMS tracker hybrids.

Despite its advantageous mass, routing density and CTE properties, the resulting substrate is very thin, flexible and is almost fully foldable with very tight angles. The integration of circuits of this kind into a tracker module, on which large quantities of wire bonds need to be appended, presents certain difficulties that need to be evaluated. The manufacturability of modules based on this type of circuits is a key issue, such as the fixation of the foil, its resulting flatness to enable the wire bonding, its thermal path properties; the gluing on a supporting and cooling structure, and simply the difficulty to handle a large assembled flexible foil with the required accuracy and without damaging it.

\section{Conclusions}

The design of the CMS tracker hybrid circuits upgrade requires flip chip ASICs in compact and low mass topologies that impose the use of very high density routing techniques. Rigid build up substrates used for chip packaging have been found to be applicable, overcoming the large board area and the number of high pin count dice. The advantage of a rigid assembly is that it can be easily tested and integrated into modules but it is balanced with the non-matching silicon coefficient of thermal expansion that could impact the system long term reliability or yield. Alternatively, flexible polyimide circuits typically developed for medical applications also meet the high density and low mass requirements. Here the very good CTE that matches the silicon one is balanced with the high flexibility of the circuit that would result in very difficult assembly and wire bonding procedures.

These types of products are compatible with any type of the CMS tracker modules. For the strip-pixelated strip modules, further developments are expected from the integration of large through silicon vias into ASICs that would enable an optimal integration of the pixel ASICs into their modules. 
On this basis, several prototypes will be developed based on these different techniques for the forthcoming design of hybrid circuits for the tracker phase 2 upgrade.

\section{References}

[1] D. Abbaneo, Upgrade of the CMS Tracker with tracking trigger, 2011 JINST 6 C12065.

[2] D. Braga et al., CBC2: a microstrip readout ASIC with coincidence logic for trigger primitives at HL-LHC, 2012 JINST 7 C10003.

[3] K. Yamanaka, K. Kobayashi, K. Hayashi and M. Fukui, Materials, processes, and performance of high-wiring density buildup substrate with ultralow-coefficient of thermal expansion, IEEE Trans. Compon. Pack. T. 33 (2010) 453.

[4] M. Friedl et al., The Belle II silicon vertex detector, in Proceedings of TIPP 2011, Chicago U.S.A., 9-14 Jun 2011 [Phys. Procedia 37 (2012) 867]. 